\title{
Noninvasive amide proton transfer magnetic resonance imaging in evaluating the grading and cellularity of gliomas
}

\author{
Yan Bai',* ${ }^{1}$, Yusong Lin ${ }^{2}{ }^{*}$, Wei Zhang ${ }^{3}$, Lingfei Kong ${ }^{4}$, Lifu Wang ${ }^{4}$, Panli Zuo ${ }^{5}$, Ignacio \\ Vallines $^{6}$, Benjamin Schmitt ${ }^{7}$, Jie Tian ${ }^{8}$, Xiaolei Song ${ }^{9}$, Jinyuan Zhou ${ }^{9}$ and Meiyun \\ Wang ${ }^{1}$ \\ ${ }^{1}$ Department of Radiology, Zhengzhou University People's Hospital \& Henan Provincial People's Hospital, Zhengzhou, Henan, \\ China \\ ${ }^{2}$ Cooperative Innovation Center of Internet Healthcare \& School of Software and Applied Technology, Zhengzhou University, \\ Zhengzhou, Henan, China \\ ${ }^{3}$ Department of Radiology, Ren Ji Hospital of Shanghai Jiao Tong University, Shanghai, China \\ ${ }^{4}$ Department of Pathology, Zhengzhou University People's Hospital \& Henan Provincial People's Hospital, Zhengzhou, Henan, \\ China \\ ${ }^{5}$ MR Collaborations NE Asia, Siemens Healthcare China, Beijing, China \\ ${ }^{6}$ MR Collaborations NE Asia, Siemens Healthcare China, Shanghai, China \\ ${ }^{7}$ Imaging \& Therapy Systems Division, Siemens Healthcare Australia, Brisbane, Australia \\ ${ }^{8}$ Institute of Automation, Chinese Academy of Sciences, Beijing, China \\ 9 Department of Radiology, Johns Hopkins University, Baltimore, MD, USA \\ * These authors have contributed equally to this work \\ Correspondence to: Meiyun Wang, email: mywang@ha.edu.cn
}

Keywords: amide proton transfer, glioma, grading, Ki-67, magnetic resonance imaging

Received: July 04, $2016 \quad$ Accepted: December 12, 2016

Published: December 15, 2016

\section{ABSTRACT}

Using noninvasive magnetic resonance imaging techniques to accurately evaluate the grading and cellularity of gliomas is beneficial for improving the patient outcomes. Amide proton transfer imaging is a noninvasive molecular magnetic resonance imaging technique based on chemical exchange saturation transfer mechanism that detects endogenous mobile proteins and peptides in biological tissues. Between August 2012 and November 2015, a total number of 44 patients with pathologically proven gliomas were included in this study. We compared the capability of amide proton transfer magnetic resonance imaging with that of noninvasive diffusion-weighted imaging and noninvasive 3-dimensional pseudo-continuous arterial spin imaging in evaluating the grading and cellularity of gliomas. Our results reveal that amide proton transfer magnetic resonance imaging is a superior imaging technique to diffusion-weighted imaging and 3-dimensional pseudo-continuous arterial spin imaging in the grading of gliomas. In addition, our results showed that the Ki-67 index correlated better with the amide proton transfer-weighted signal intensity than with the apparent diffusion coefficient value or the cerebral blood flow value in the gliomas. Amide proton transfer magnetic resonance imaging is a promising method for predicting the grading and cellularity of gliomas.

\section{INTRODUCTION}

Glioma is the most common primary tumor in the brain and is classified into four grades based on the World Health Organization (WHO) guidelines [1]. Accurate grading of a glioma is fundamental in order to determine the treatment strategy. Ki-67 index is a biomarker of cellularity in gliomas, which is elevated by increasing cellular density [2]. Using noninvasive magnetic resonance imaging (MRI) techniques to accurately evaluate the grading and cellularity of gliomas is beneficial for improving the patient outcomes. 
Table 1: The APTw signal intensity, ADC value, CBF value and Ki-67 index (mean \pm standard deviation) in the WHO grade 2,3 and 4 gliomas

\begin{tabular}{lcccc}
\hline & $\begin{array}{c}\text { APTw signal } \\
(\%)\end{array}$ & $\begin{array}{c}\text { ADC } \\
\left(10^{-3} \mathrm{~s} / \mathrm{mm}^{2}\right)\end{array}$ & $\begin{array}{c}\text { CBF } \\
(\mathrm{ml} / 100 \mathrm{~g} / \mathrm{min})\end{array}$ & Ki-67 \\
\hline $\begin{array}{l}\text { WHO grade 2 gliomas } \\
(\mathrm{n}=18)\end{array}$ & $1.25 \pm 0.17$ & $1.24 \pm 0.24$ & $58.3 \pm 8.2$ & $0.19 \pm 0.11$ \\
$\begin{array}{l}\text { WHO grade 3 gliomas } \\
\quad(\mathrm{n}=10)\end{array}$ & $1.71 \pm 0.45$ & $1.04 \pm 0.24$ & $63.9 \pm 19.6$ & $0.36 \pm 0.12$ \\
$\begin{array}{l}\text { WHO grade 4 gliomas } \\
(\mathrm{n}=16)\end{array}$ & $2.05 \pm 0.18$ & $1.05 \pm 0.16$ & $69.2 \pm 18.6$ & $0.61 \pm 0.12$ \\
$\begin{array}{l}P \text { values between WHO } \\
\text { grade 2 and 3 glimas }\end{array}$ & $0.005^{*}$ & 0.024 & 0.874 & $0.001^{*}$ \\
$P$ values between WHO & $<0.001^{*}$ & $0.014^{*}$ & 0.023 & $<0.001^{*}$ \\
$\begin{array}{l}\text { grade 2 and 4 glimas } \\
P \text { values between WHO } \\
\text { grade 3 and 4 glimas }\end{array}$ & $0.015^{*}$ & 0.461 & 0.213 & $<0.001^{*}$ \\
\hline
\end{tabular}

Abbreviations: APTw, amide proton transfer-weighted; ADC, apparent diffusion coefficient; $\mathrm{CBF}$, cerebral blood flow; WHO, World Health Organization. * Values are significant with Bonferroni corrections for multiple comparisons $(P<0.016)$.

Amide proton transfer (APT) imaging is a noninvasive molecular MRI technique based on chemical exchange saturation transfer mechanism that detects endogenous mobile proteins and peptides in biological tissues [3-5]. Preliminary studies have shown that APTweighted (APTw) signal intensity could serve as a new imaging biomarker, by revealing significantly higher signal intensities in the high-grade gliomas compared with the low-grade gliomas [6-9].

Diffusion-weighted imaging (DWI) is a noninvasive MRI technique to describe the diffusion of water molecules. The apparent diffusion coefficient (ADC) derived from DWI has been widely used to grade gliomas and reflects tumor cellularity $[10,11]$. However, ADC is not reliable enough for assessing the grading and cellular density of gliomas [12-14].

3-dimensional (3D) pseudo-continuous arterial spin labeling (pCASL) imaging is a noninvasive perfusion MRI method that allows quantification of cerebral blood flow (CBF) by using magnetic tagging of the arterial blood-water protons as an endogenous tracer. Currently, the pCASL tagging method provides the best signal-tonoise ratio [15]. The previous study has been used the 3D pCASL MRI method to evaluate the grading of gliomas [16].

To the best of our knowledge, no previous study has investigated whether APT MRI could improve the predictions of grading and cellularity in gliomas compared with DWI and 3D pCASL imaging. The purpose of this study was to compare the capability of APT MRI with that of DWI and 3D pCASL imaging in evaluating the grading and cellularity of gliomas.

\section{RESULTS}

Table 1 quantitatively lists the APTw signal intensity, $\mathrm{ADC}$ value, $\mathrm{CBF}$ value and $\mathrm{Ki}-67$ index among the WHO grade 2, 3 and 4 gliomas. Table 2 lists the information of included patients. Figures 1,2 and 3 showed the manifestations of WHO grade 2, 3 and 4 gliomas on T1-weighted (T1w) images, T2-weighted (T2w) images, post-gadolinium T1w images, ADC maps, CBF maps, APT maps and Ki-67 immunohistostaining maps, respectively. Figure 4 showed the box and whisker plots of the APTw signal intensity (Figure 4A), ADC value (Figure 4B) and $\mathrm{CBF}$ value (Figure $4 \mathrm{C}$ ) in the WHO grade 2, 3 and 4 gliomas.

After Bonferroni corrections for multiple comparisons, the APTw signal intensities were significantly different between the WHO grade 2 and 3, 2 and 4 , and 3 and 4 gliomas ( $U=32,1$ and 24, respectively; $P=0.005, P<0.001$ and $P=0.015$, respectively). The ADC values were significant higher in the $\mathrm{WHO}$ grade 2 gliomas than those in the WHO grade 4 gliomas $(U=73$, $P=0.014$ ), whereas there were no significant differences between the WHO grade 2 and 3 , and 3 and 4 gliomas $(U=43$ and 66, respectively; $P=0.024$ and 0.461 , respectively). The $\mathrm{CBF}$ values were not significantly different between the WHO grade 2 and 3, 2 and 4, and 3 and 4 gliomas ( $U=77,78$ and 64, respectively; $P=0.874$, 0.023 and 0.213 , respectively). The Ki-67 indexes were significantly different between the WHO grade 2 and 3, 2 and 4 , and 3 and 4 gliomas ( $U=27,3$ and 11 , respectively; $P=0.001, P<0.001$ and $P<0.001$, respectively).

There was a significant correlation coefficient between the APTw signal intensity and the Ki-67 index ( $\mathrm{r}$ $=0.597$ [0.357 to 0.781 with $95 \%$ confidence interval], $P<$ 


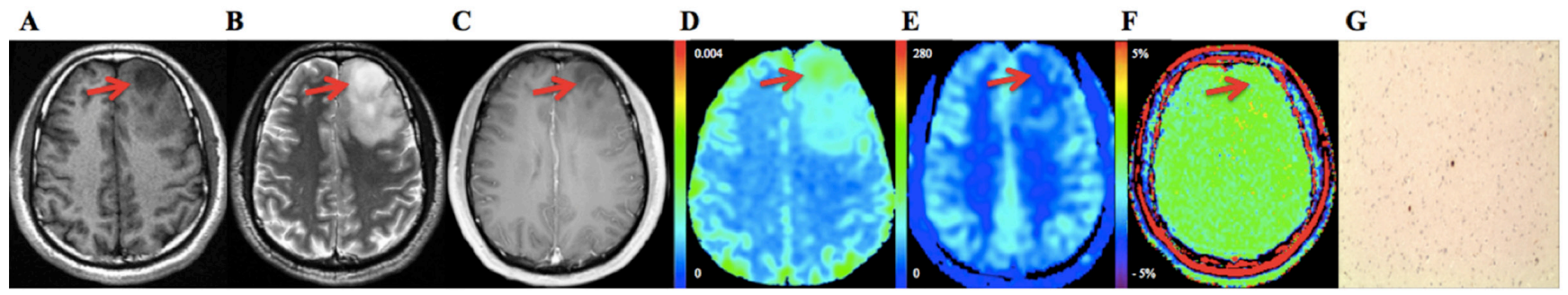

Figure 1: A 25-year-old male patient with astrocytoma (WHO grade 2) in the left frontal lobe. The tumor has peritumoral edema. The solid tumor components (arrows) show hypointense signals on T1w image A. and hyperintense signals on T2w image B. No enhancement is revealed on post-gadolinium T1w image C. ADC map D. shows increased value. Both CBF map E. and APT map F. demonstrate no increasements of the values. Ki-67 immunohistostaining map G. shows no obvious increasement of the expression (original magnification, $\times 100)$.

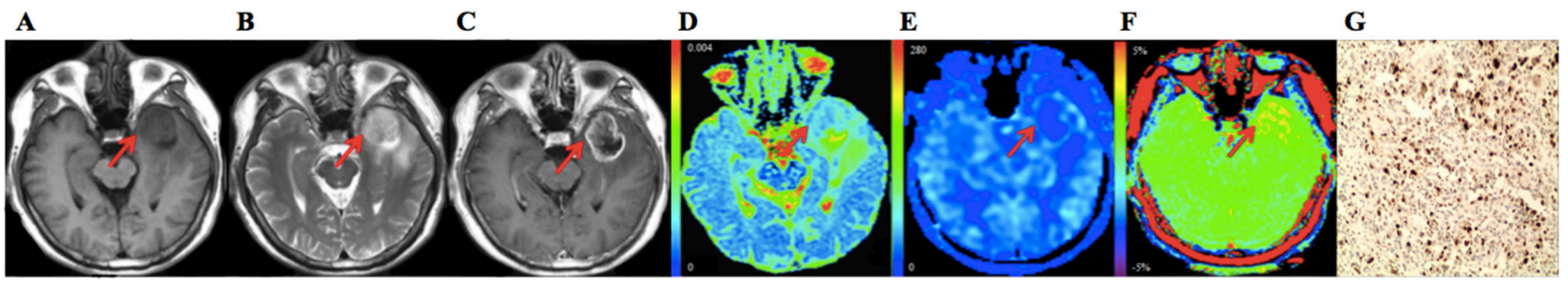

Figure 2: A 52-year-old female patient with astrocytoma (WHO grade 3) in the left temporal lobe. The tumor (arrows) shows hypointense signals on T1w image A. and heterogeneous hyperintense signals on T2w image B. Post-gadolinium T1w image demonstrates an irregular ring-enhanced tumor with central no enhancement $\mathbf{C}$. ADC map D. shows heterogeneous values. CBF map E. shows ring increased values and no increasement of the value in the central tumor. APT map F. demonstrates heterogeneous increased values. Ki-67 immunohistostaining map G. shows increasement of the expression (original magnification, $\times 100$ ).

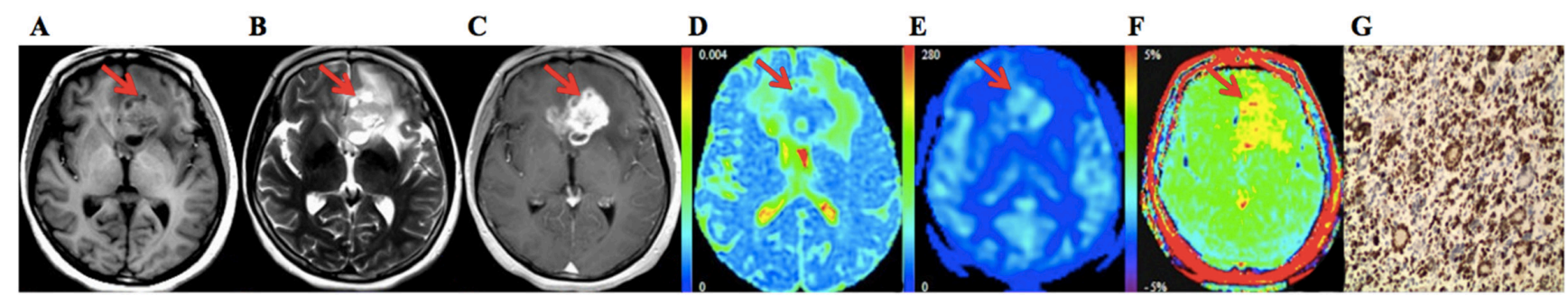

Figure 3: A 59-year-old male patient with glioblastoma (WHO 4 grade) in the left frontal lobe. The tumor (arrows) shows heterogeneous hypointense signals on T1w image A. and heterogeneous hyperintense signals on T2w image B.Heterogeneous enhancement is noted on post-gadolinium T1w image C. ADC map D. shows decreased values. Both CBF map E. and APT map F. demonstrate increased values. Ki-67 immunohistostaining map G. shows obvious increasement of the expression (original magnification, $\times 100$ ).

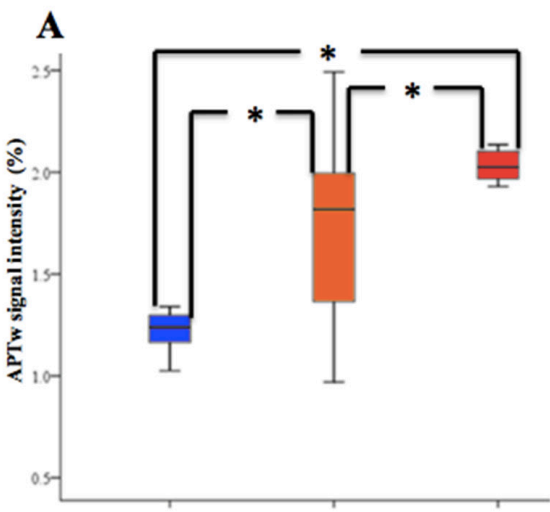

WHO grade 2 WHO grade 3 WHO grade 4

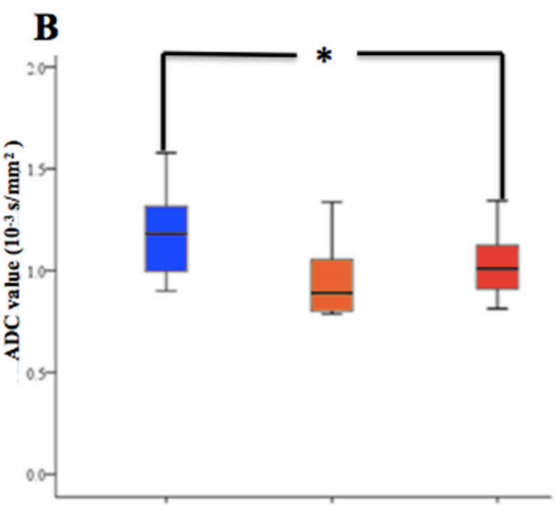

WHO grade 2 WHO grade 3 WHO grade 4

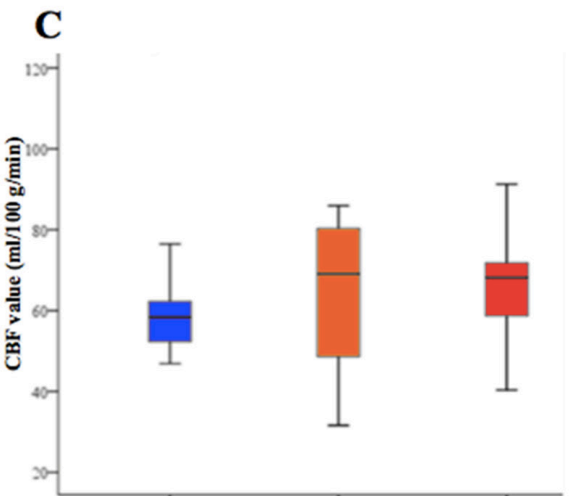

WHO grade 2 WHO grade 3 WHO grade 4

Figure 4: Box and whisker plots of the APTw signal intensity A., ADC value B. and CBF value C. in the WHO grade 2, 3 and 4 gliomas. * Values are significant with Bonferroni corrections for multiple comparisons $(P<0.016)$. 
Table 2: The information of included patients

\begin{tabular}{|c|c|c|c|c|c|c|}
\hline $\begin{array}{l}\text { Document } \\
\text { number }\end{array}$ & Gender & $\begin{array}{l}\text { Age } \\
\text { (years) }\end{array}$ & \begin{tabular}{|l|} 
Grade \\
(WHO \\
criteria)
\end{tabular} & Histology & $\begin{array}{l}\text { Tumor size } \\
\text { Maximal cross-sectional } \\
\text { diameters }(\mathrm{mm} \times \mathbf{m m})\end{array}$ & Tumor location \\
\hline 1 & Female & 38 & 2 & astrocytoma & $53 \times 49$ & Right frontal lobe \\
\hline 2 & Female & 57 & 4 & glioblastoma & $67 \times 62$ & Right temporal lobe \\
\hline 3 & Male & 59 & 4 & glioblastoma & $54 \times 41$ & Left frontal lobe \\
\hline 4 & Female & 52 & 3 & oligoastrocytoma & $42 \times 50$ & Right temporal lobe \\
\hline 5 & Female & 62 & 4 & glioblastoma & $45 \times 52$ & Left frontal lobe \\
\hline 6 & Male & 48 & 4 & glioblastoma & $36 \times 36$ & Right parietal lobe \\
\hline 7 & Male & 43 & 2 & astrocytoma & $41 \times 44$ & Left temporal lobe \\
\hline 8 & Female & 46 & 3 & oligoastrocytoma & $42 \times 41$ & Left parietal lobe \\
\hline 9 & Male & 63 & 3 & oligodendroglioma & $53 \times 64$ & Right frontal lobe \\
\hline 10 & Male & 54 & 4 & glioblastoma & $46 \times 43$ & Right frontal lobe \\
\hline 11 & Male & 68 & 2 & oligodendroglioma & $52 \times 49$ & Right parietal lobe \\
\hline 12 & Male & 57 & 4 & glioblastoma & $42 \times 39$ & Left temporal lobe \\
\hline 13 & Female & 58 & 4 & glioblastoma & $47 \times 43$ & Left temporal lobe \\
\hline 14 & Male & 54 & 4 & glioblastoma & $34 \times 37$ & Right parietal lobe \\
\hline 15 & Male & 25 & 2 & astrocytoma & $34 \times 31$ & Left frontal lobe \\
\hline 16 & Male & 66 & 4 & glioblastoma & $43 \times 40$ & Left occipital lobe \\
\hline 17 & Female & 47 & 3 & oligoastrocytoma & $42 \times 56$ & Right occipital lobe \\
\hline 18 & Female & 52 & 3 & astrocytoma & $33 \times 36$ & Left temporal lobe \\
\hline 19 & Female & 50 & 2 & oligoastrocytoma & $45 \times 51$ & Right parietal lobe \\
\hline 20 & Male & 49 & 4 & glioblastoma & $39 \times 53$ & Right temporal lobe \\
\hline 21 & Male & 49 & 4 & glioblastoma & $61 \times 54$ & Right parietal lobe \\
\hline 22 & Female & 41 & 2 & oligoastrocytoma & $38 \times 43$ & Left frontal lobe \\
\hline 23 & Female & 40 & 2 & astrocytoma & $22 \times 27$ & Left temporal lobe \\
\hline 24 & Male & 35 & 2 & astrocytoma & $47 \times 42$ & Right temporal lobe \\
\hline 25 & Male & 60 & 4 & glioblastoma & $47 \times 51$ & Left occipital lobe \\
\hline 26 & Male & 34 & 2 & astrocytoma & $26 \times 31$ & Right frontal lobe \\
\hline 27 & Male & 43 & 3 & oligodendroglioma & $58 \times 72$ & Left frontal lobe \\
\hline 28 & Female & 27 & 2 & oligoastrocytoma & $48 \times 52$ & Right parietal lobe \\
\hline 29 & Male & 63 & 4 & glioblastoma & $68 \times 63$ & Right temporal lobe \\
\hline 30 & Male & 37 & 3 & astrocytoma & $64 \times 59$ & Left temporal lobe \\
\hline 31 & Female & 54 & 2 & astrocytoma & $38 \times 41$ & Left temporal lobe \\
\hline 32 & Female & 50 & 2 & astrocytoma & $36 \times 34$ & Right frontal lobe \\
\hline 33 & Male & 41 & 4 & glioblastoma & $39 \times 53$ & Left parietal lobe \\
\hline 34 & Female & 29 & 2 & astrocytoma & $34 \times 34$ & Right parietal lobe \\
\hline 35 & Female & 45 & 2 & astrocytoma & $49 \times 47$ & Right temporal lobe \\
\hline 36 & Female & 68 & 3 & astrocytoma & $42 \times 49$ & Left temporal lobe \\
\hline 37 & Male & 38 & 2 & astrocytoma & $31 \times 34$ & Left frontal lobe \\
\hline 38 & Female & 69 & 4 & glioblastoma & $46 \times 48$ & Left frontal lobe \\
\hline 39 & Female & 51 & 2 & oligodendroglioma & $41 \times 36$ & Left parietal lobe \\
\hline 40 & Female & 56 & 2 & astrocytoma & $52 \times 57$ & Right temporal lobe \\
\hline 41 & Male & 65 & 3 & astrocytoma & $51 \times 44$ & Left parietal lobe \\
\hline 42 & Female & 51 & 2 & astrocytoma & $22 \times 32$ & Left frontal lobe \\
\hline 43 & Female & 45 & 4 & glioblastoma & $42 \times 40$ & Left temporal lobe \\
\hline 44 & Female & 44 & 3 & astrocytoma & $50 \times 47$ & Right frontal lobe \\
\hline
\end{tabular}

0.001), and between the ADC value and the Ki-67 index ( $\mathrm{r}$ $=-0.441$ [ -0.669 to -0.179 with $95 \%$ confidence interval], $P=0.003)$ in the gliomas. Moreover, the correlation coefficient between the APTw signal intensity and the Ki-67 index was significantly higher than that between the ADC value and the Ki-67 index in the gliomas $(P<$
0.01) (Figure 5A). The CBF value was not significantly correlated with the Ki-67 index $(r=0.245[-0.045$ to 0.516 with $95 \%$ confidence interval], $P=0.109$ ) in the gliomas (Figure 5B).

The areas under the receiver operating characteristic (ROC) curves (AUCs) were analyzed as measures for the 
grading of gliomas. The AUC of the APTw signal intensity ( $0.997 ; 0.890$ to 1.000 with $95 \%$ confidence interval) was significantly greater than that of the ADC value $(0.745$; 0.567 to 0.878 with $95 \%$ confidence interval $)(P=0.002)$ and the $\mathrm{CBF}$ value $(0.729 ; 0.550$ to 0.866 with $95 \%$ confidence interval) $(P=0.002)$ in the differentiation of the WHO grade 2 and 4 glimas (Figure 6A). There were no significantly differences between the AUCs of the APTw signal intensity $(0.825 ; 0.635$ to 0.941 with $95 \%$ confidence interval) and the ADC value $(0.767 ; 0.569$ to 0.904 with $95 \%$ confidence interval) $(P>0.05)$, and between the AUCs of the APTw signal intensity and the CBF value $(0.644 ; 0.442$ to 0.815 with $95 \%$ confidence interval) $(P>0.05)$ for the grading of WHO grade 2 and 3 gliomas (Figure 6B). For discriminating the WHO grade 3 gliomas from the WHO grade 4 gliomas, the AUC of the APTw signal intensity $(0.788 ; 0.584$ to 0.921 with $95 \%$ confidence interval) was significantly greater than that of the CBF value $(0.481 ; 0.283$ to 0.684 with $95 \%$ confidence interval) $(P=0.029)$, while there were no significantly differences between the AUCs of the APTw signal intensity and the ADC value $(0.584 ; 0.376$ to 0.772 with $95 \%$ confidence interval) $(P>0.05)$ (Figure $6 \mathrm{C})$.

The intraclass correlation coefficients between two independent radiologists (Y.B. and M.W., with 9 and 18 years of experience, respectively) for the calculations of the APTw signal intensities, ADC values and CBF values were $0.919,0.845$ and 0.909 , respectively. The intraclass correlation coefficient between two independent pathologists (L.W. and L.K., with 14 and 23 years of experiences, respectively) for the calculations of the $\mathrm{Ki}$ 67 indexes was 0.874 .
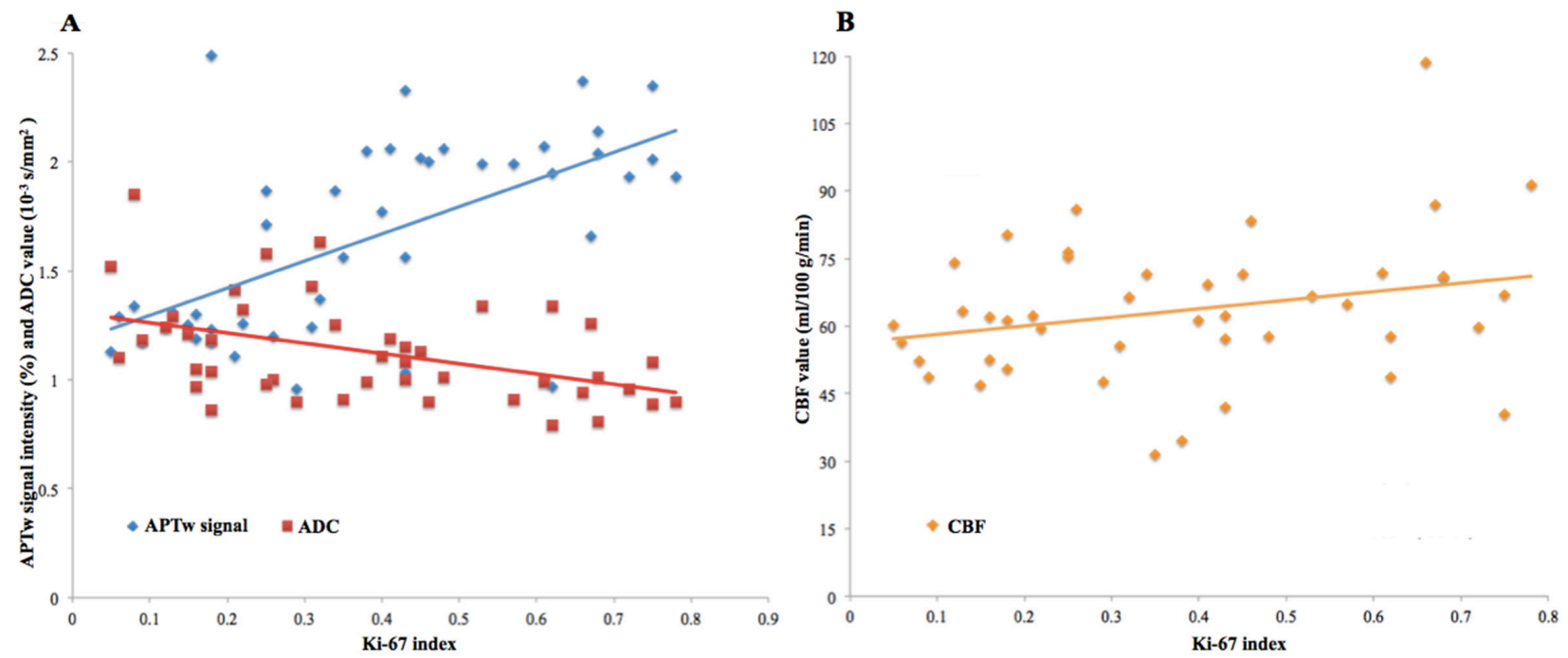

Figure 5: A. The correlation coefficient between the APTw signal intensity and the Ki-67 index $(r=0.597, P<0.001)$ is significantly higher than that between the ADC value and the Ki-67 index $(r=\mathbf{- 0 . 4 4 1 , P = 0 . 0 0 3 )}$ in the gliomas $(P<0.01)$. B. The CBF value is not significantly correlated with the Ki-67 index $(r=0.245, P=0.109)$ in the gliomas.
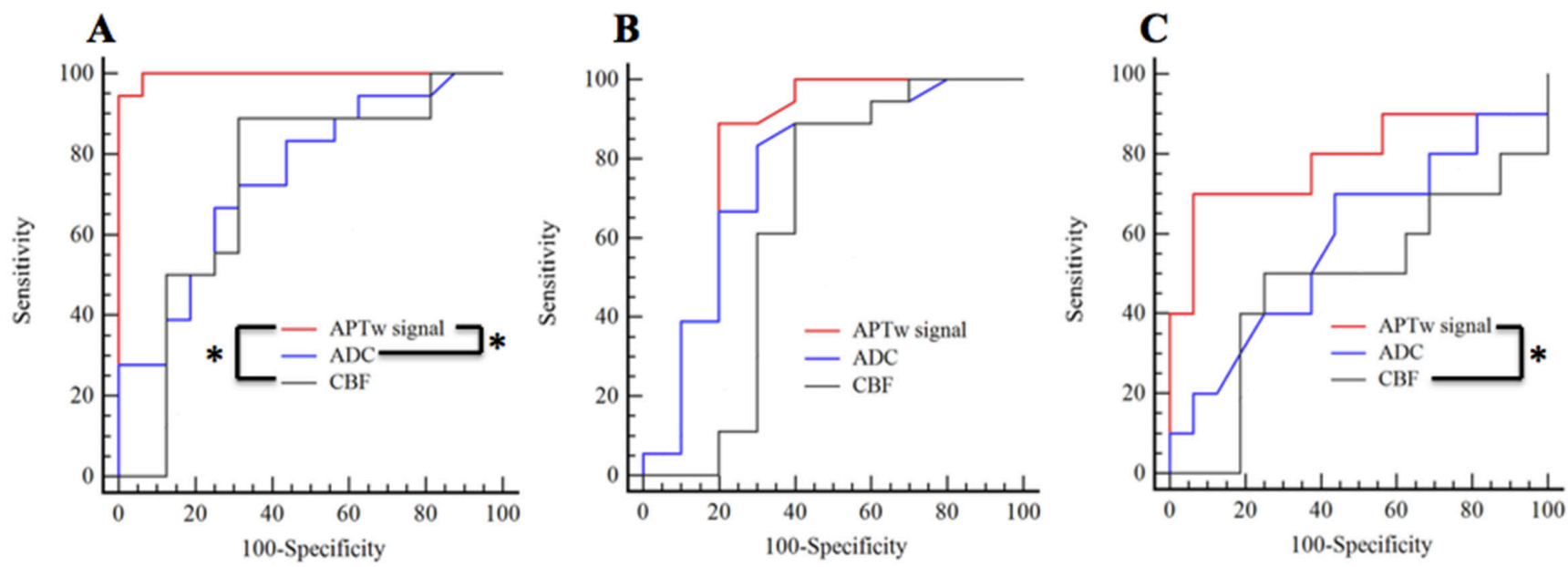

Figure 6: ROC curves for the APTw signal, ADC and CBF in distinguishing WHO grade 2 gliomas from WHO grade 4 gliomas A., WHO grade 2 gliomas from WHO grade 3 gliomas B. and WHO grade 3 gliomas from WHO grade 4 gliomas C. * Values are significant $(P<0.05)$. 


\section{DISCUSSION}

In our present study, the results showed that the APTw signal intensity was significantly different between the WHO grades 2, 3 and 4 gliomas. However, the ADC value and $\mathrm{CBF}$ value had inconsistently significant differences between the WHO grades 2, 3 and 4 gliomas. In addition, our results demonstrated that the Ki-67 index correlated better with the APTw signal intensity than with the $\mathrm{ADC}$ value or the $\mathrm{CBF}$ value in the gliomas.

Our results support that APT MRI is a valuable tool in evaluating the grading and cellularity of gliomas. Previous studies reported that the high-grade gliomas had higher concentration of mobile proteins and peptides than the low-grade gliomas [6-9]. In addition, APT MRI can provide contrast based on the cellular mobile proteins and peptides $[3,4]$. Thus, APTw signal intensity has the capability to represent the cellular density of gliomas [7]. Park et al. [8] consider that APT MRI is a promising in vivo imaging method for quantifying the cellular proliferation of gliomas. The higher APTw signal intensity in the higher-grade gliomas may be attributed to the denser cellularity in the solid components of these tumors in comparison with the lower-grade gliomas. It is worth noting that the APTw contrast visualized at around $3.5 \mathrm{ppm}$ may include other contributions beside mobile proteins and peptides such as the effect of $\mathrm{pH}$ on proton exchange and nuclear Overhauser effect (NOE) [4, 17]. The $\mathrm{pH}$ environment changes the amide proton exchange rate, which influences the APTw contrast [3]. However, the effect of $\mathrm{pH}$ on the APTw signal intensities of gliomas should be negligible because only minimally increased intracellular $\mathrm{pH}$ was detected in gliomas in comparison to the normal brain tissues [18]. NOE signal entangled APTw contrast in MTR asymmetry analysis by lower signal in tumor at the unfiled of the water resonance $(-2.5$ to $-5 \mathrm{ppm})$. However, the NOE signal can be clearly detected at lower saturation powers, while the APT signal is maximized at relatively higher saturation powers. In this study, the average saturation power of $2.0 \mu \mathrm{T}$ was used to minimize the NOE effects for APT MRI [17]. In addition, the NOE effect is usually ignorable at 3-T MRI, but the effect is very high at 7-T MRI [17]. Furthermore, the cystic changes within the gliomas contain many mobile proteins and show high APTw signal intensities, which may lead to errors [6]. Therefore, we delineated the ROIs without the areas of necrosis and cystic degeneration on the $\mathrm{T} 2 \mathrm{w}$ images.

In addition, our results showed that the $\mathrm{ADC}$ value was significantly different between the WHO grade 2 and 4 gliomas. This finding is consistent with some of the previous studies demonstrating that the $\mathrm{ADC}$ value representing cellularity of gliomas is significantly lower in the high-grade gliomas than that in the low-grade gliomas $[10,11]$. However, our current results also showed that there were no differences in the ADC values between the
WHO grade 2 and 3, and grade 3 and 4 gliomas. Indeed, some previous reports have demonstrated no significant difference in the ADC values between the high-grade and low-grade gliomas $[12,19]$. Moreover, Sadeghi et al. [13] reported that the ADC value was not correlated with the cellular density of gliomas. One possible explanation for those contradictory results is that the higher tumor cellularity and vascularity in the higher-grade gliomas [11, 19] may affect the DWI signal attenuation in an opposite way [20-22]. Therefore, the ADC value has limitations in evaluating the grading and cellularity of gliomas.

The results of our present study demonstrated that there were no differences in the $\mathrm{CBF}$ values obtained from 3D pCASL MRI between the WHO grade 2, 3, and 4 gliomas. Our finding was consistent with the previous study reported by Roy et al. [16], which showed that 3D pCASL MRI could not be regarded as a reliable technique in the grading of gliomas. The CBF value quantified by pCASL method was influenced by a number of factors such as arterial transit time, inversion efficiency and the magnitude of the error [16]. These unsatisfied results may be caused by the inaccurate quantifications of CBF values [16]. Moreover, the $\mathrm{CBF}$ value could not represent the cellularity of gliomas.

There are some limitations in our study. First, the patient population was relatively small. Second, every grade gliomas contained different pathological subtypes, which would cause statistical deviations. In addition, the APT MRI used in this study was a 2-dimensional (2D) single-slice approach, which could not provide coverage of the whole tumor. In the future, we will enroll more patients with gliomas and investigate the differences between different pathological subtypes. Moreover, the APT MRI in monitoring the tumor recurrence and the treatment response should be investigated further.

In conclusion, APT MRI may be a superior imaging technique to DWI and 3D pCASL imaging in evaluating the grading and cellularity of gliomas. APT MRI is a promising method for predicting the grading and cellularity of gliomas.

\section{MATERIALS AND METHODS}

\section{Patient population}

A total number of 51 patients with pathologically proven gliomas were enrolled between August 2012 and November 2015 in this study. The inclusion criteria included: (a) MRI examinations performed on patients prior to treatments of gliomas; (b) the pathological diagnoses and Ki-67 indexes were acquired by surgical resections of gliomas and (c) APT MRI was performed at least 24 hours after the injection of contrast agents to minimize the influence of the gadopentetate dimeglumine 
in the APTw signal intensity [23]. The exclusion criteria included: (a) poor imaging quality due to motion artifacts; (b) solid tumor component unavailable for analysis ( $<20 \mathrm{~mm}^{2}$ ) and (c) WHO grade 1 gliomas because they were distinctive in the population (pediatric) and location (often posterior fossa) [24]. Finally, 5 patients with head movement artifacts and 2 patients with unavailable solid tumor components were excluded. A total number of 44 patients (21 males and 23 females; age range, 25-68 years; mean age \pm standard deviation, $49 \pm 11$ years) with gliomas were included in the statistical analyses. Out of the 44 patients, 18 patients (41\%) were confirmed with pathology to be WHO grade 2 gliomas, 10 patients (23\%) were WHO grade 3 gliomas, and the remaining 16 patients $(36 \%)$ were WHO grade 4 gliomas. The diagnoses of the patients included WHO grade 2 astrocytomas $(n=13)$, grade 2 oligodendrogliomas $(n=2)$, grade 2 oligoastrocytomas $(n=3)$, grade 3 anaplastic astrocytomas $(n=5)$, grade 3 anaplastic oligodendrogliomas $(n=2)$, grade 3 anaplastic oligoastrocytomas $(n=3)$, and grade 4 glioblastomas $(n=16)$.

This study was approved by the institutional review board of Zhengzhou University People's Hospital \& Henan Provincial People's Hospital, and written informed consent was obtained from each subject before participation.

\section{MRI data acquisition}

All patients were examined using conventional MRI, DWI and 3D pCASL imaging in a 3-T MRI unit (Discovery MR 750, General Electric Medical Systems, Milwaukee, Wisconsin, USA) with an eight-channel head coil (General Electric Medical System). Conventional fast spin-echo (FSE) sequences were performed including axial T1w image with a repetition time (TR) of $1593 \mathrm{~ms}$, an echo time (TE) of $24 \mathrm{~ms}$, a field of view (FOV) of 24 $\times 24 \mathrm{~cm}^{2}$, a matrix of $320 \times 256$ and a slice thickness of $4 \mathrm{~mm}$, and axial T2w image (TR/TE, $4600 \mathrm{~ms} / 110 \mathrm{~ms}$; FOV, $24 \times 24 \mathrm{~cm}^{2}$; matrix, $320 \times 256$; and slice thickness, $4 \mathrm{~mm}$ ). The axial $\mathrm{T} 1 \mathrm{w}$ sequence was repeated after intravenous administration of $0.01 \mathrm{mmol} / \mathrm{kg}$ gadopentetate dimeglumine (Magnevist, Bayer Schering Pharma, Berlin, Germany). DWI and 3D pCASL imaging were performed before the injection of contrast agents. DWI was obtained using a single-shot, echo-planar sequence in the axial plane (TR/TE, $4000 \mathrm{~ms} / 112 \mathrm{~ms}$; FOV, $24 \times 24 \mathrm{~cm}^{2}$; matrix, $128 \times 128$; slice thickness, $4 \mathrm{~mm}$; and slice number, 38). Two $b$ values of 0 and $1000 \mathrm{~s} / \mathrm{mm}^{2}$ (1 signal acquisition) were used in three orthogonal directions. The acquisition time for DWI was 48 seconds. 3D pCASL MRI was acquired using a FSE sequence in the axial plane (TR/TE, $4623 \mathrm{~ms} / 10.5 \mathrm{~ms}$; FOV, $24 \times 24 \mathrm{~cm}^{2}$; slice thickness, 4 $\mathrm{mm}$; slice number, 38; labeling duration, $2025 \mathrm{~ms}$; and post-labeling delay time, $1525 \mathrm{~ms}$ ). A total of 8 arms were acquired and each spiral arm included 512 sampling points in the k-space. The acquisition time for 3D pCASL MRI was 4 minutes and 24 seconds.

All patients were also examined using a prototype 2D single-slice radiofrequency-spoiled gradient echo APT MRI protocol in a 3-T MRI unit (Magnetom Trio, Siemens Healthcare, Erlangen, Germany) with a twelvechannel head coil (Siemens Healthcare). The APT MRI was obtained with a TR of $3200 \mathrm{~ms}$, a TE of $2.87 \mathrm{~ms}$, a FOV of $24 \times 24 \mathrm{~cm}^{2}$, a matrix of $128 \times 128$, a slice thickness of $4 \mathrm{~mm}, 21$ frequency offsets from -5 to +5 ppm with even intervals of $0.5 \mathrm{ppm}$. The unsaturated water image was acquired as first image in the APT MRI series. The presaturation was achieved using a train of five Gaussian-shaped radiofrequency saturation pulses with a continuous-wave amplitude equivalent of 2.0 $\mu \mathrm{T}$. The total duration of saturation time was $995 \mathrm{~ms}$ followed by the readout of a single gradient echo image with centric reordering. The length of the each saturation radiofrequency pulse was $99 \mathrm{~ms}$, and the gap between the pulses was $100 \mathrm{~ms}$. The acquisition time was 1 minute and 45 seconds for a single slice. For each patient, two different slices of APT MRI were positioned at the same slice positions as the $\mathrm{T} 2 \mathrm{w}$ images selected by the two radiologists.

\section{MRI data processing}

Both DWI and 3D pCASL data were obtained and transferred to a workstation (Advantage Workstation 4.5, General Electric Medical System, Milwaukee, Wisconsin, USA) for processing.

The ADC value was calculated from the $b$ values of 0 and $1000 \mathrm{~s} / \mathrm{mm}^{2}$ by a mono-exponential model as follow [20]:

$\mathrm{S}(\mathrm{b}) / \mathrm{S}(0)=\exp (-\mathrm{b} \times \mathrm{ADC})$,

where $\mathrm{S}(\mathrm{b})$ represents the signal intensity in the presence of diffusion sensitization, and $S(0)$ represents the signal intensity in the absence of diffusion sensitization.

The CBF value was calculated by the following equation [25]:

$$
\mathrm{CBF}=\frac{\lambda\left(1-e^{\frac{-t_{s a t}}{T_{1} g}}\right)}{2 \alpha T_{1 b}\left(1-e^{\frac{-\tau}{T_{1 b}}}\right)} \frac{P W}{P D} e^{\frac{-P L D}{T_{1 b}}}
$$

where $\mathrm{t}_{\text {sat }}$ represents the time of saturation performed before imaging $(2000 \mathrm{~ms}), \mathrm{T}_{1 \mathrm{~g}}$ represents the $\mathrm{T}_{1}$ of the gray matter $(1200 \mathrm{~ms})$, and $\mathrm{T}_{1 \mathrm{~b}}$ represents the $\mathrm{T}_{1}$ of the blood (1600 ms). $\alpha$ is the labeling efficiency $(0.8), \lambda$ is the cortex-blood partition coefficient $(0.9)$, and $\tau$ is the labeling duration. PW means the perfusion-weighted map, and PD means the proton density map. PLD represents the post-labeling delay time.

The APT data processing procedures were performed by Interactive Data Language (IDL, Research Systems, Inc., Boulder, Colorado, USA). The APT images were calculated by the magnetization transfer asymmetry between signal intensities of $\pm 3.5 \mathrm{ppm}$ with respect to the 
water frequency using $\mathrm{B}_{0}$ corrected $\mathrm{z}$-spectrum on a pixelby-pixel basis [5]. The APTw value was calculated by the following equation [3]:

$\operatorname{MTR}_{\text {asym }}(3.5 \mathrm{ppm})=\operatorname{MTR}(+3.5 \mathrm{ppm})-\operatorname{MTR}(-3.5$ $\mathrm{ppm})=\mathrm{S}_{\text {sat }}(-3.5 \mathrm{ppm}) / \mathrm{S}_{0}-\mathrm{S}_{\text {sat }}(+3.5 \mathrm{ppm}) / \mathrm{S}_{0}$

Where MTR $_{\text {asym }}$ represents the magnetization transfer ratio asymmetry. MTR represents the magnetization transfer ratio. $\mathrm{S}_{\text {sat }}$ and $\mathrm{S}_{0}$ represent the signal intensities measured with and without radiofrequency saturation, respectively.

The raw image data were organized into the z-spectrum, and then the z-spectrum was fitted using a 12 th-order polynomial on a voxel-by-voxel basis. The unsaturated water image was used as a reference to calculate the APT effect. The $\mathrm{B}_{0}$ field inhomogeneity was calculated according to the deviation of the minimum of the fitted curve from $0 \mathrm{ppm}$. The correction of $\mathrm{B}_{0}$ field inhomogeneity in the APT MRI data-processing was used to make the results more reliably.

\section{MRI data analysis}

The two radiologists, who were blinded to the pathological results of the tumors, independently analyzed all the APT, ADC and CBF maps by using Image J software (version 1.49b, NIH, USA). Before drawing the regions of interest (ROIs), the matrixes of T2w and CBF images were changed to $128 \times 128$. The two radiologists independently drew ROIs on the selected axial T2w images for each tumor. The ROIs were manually drawn around the entire solid part of the tumors. Areas of necrosis, cystic component, edema, cerebrospinal fluid or hemorrhage were excluded to ensure accurate measurements. Then, the ROIs on T2w images were copied to the APT, ADC and CBF maps of the same patient for the measurements.

\section{Histological processing immunohistochemistry}

Specimens acquired from surgical resections were embedded in paraffin. Ki-67 immunohistostaining was conducted for quantification analysis. Slides were rinsed in phosphate buffer saline and blocked with $5 \%$ normal goat serum, followed by incubation with primary mouse monoclonal mouse anti-Ki-67 (ZM0165, Zhongshan Biotechnology Co., Ltd., Beijing, China) for 2 hours at $37^{\circ} \mathrm{C}$. Slides were then incubated with horseradish peroxidase-conjugated secondary antibody diaminobenzidine (Fuzhou Maixin Biotechnology Development Co., Ltd., Fuzhou, Fujian, China) for 10 minutes at $37^{\circ} \mathrm{C}$, and visualized with diaminobenzidine substrate (Fuzhou Maixin Biotechnology Development Co., Ltd., Fuzhou, Fuzhou, China).

\section{Immunohistochemistry data analysis}

The two pathologists independently analyzed the Ki67 index by using a HMIAS-2000 Medical Color Image Analysis System (Champion Image Engineering Co., Ltd., Wuhan, Hubei, China). They independently placed three different ROIs in the solid components of the tumor on the Ki-67 immunohistostaining map for each patient. The ROIs excluded the areas of necrosis, cystic degeneration or hemorrhage. The integrated optical density was measured for the Ki-67 index.

\section{Statistical analysis}

All statistical analyses were performed with SPSS software (version 17.0, SPSS, Chicago, Ill). The mean results of APTw signal intensity, ADC value and $\mathrm{CBF}$ value for each patient measured by the two radiologists and the mean result of Ki-67 index for each subject measured by the two pathologists were used for statistical analyses. A Mann-Whitney $U$ test was used for the comparison of each MRI parameter and Ki-67 index between the WHO grade 2, 3 and 4 gliomas. Bonferroni corrections were used for multiple comparisons. Spearman rank correlation analyses with $95 \%$ confidence intervals were used for assessing correlation coefficients between each MRI parameter and Ki-67 index in the gliomas. A Fisher's z transformation analysis was used for comparing the correlation coefficients. A ROC curve analysis with $95 \%$ confidence interval was used to assess the diagnostic utilities of APTw signal intensity, ADC value and CBF value in the grading of gliomas. The interobserver agreement for each quantitative measurement was assessed by using the intraclass correlation coefficient.

\section{GRANT SUPPORT}

This work was supported by National Natural Science Foundation of China (81271565, 31470047 and 81601466), National Institutes of Health (R21-EB02090501A1), Henan Province Scientific and Technological Innovation Talents Project (164200510014), Cooperation Project (152106000014) and Key Project of Henan Medical Science and Technology Project (201501011).

\section{CONFLICTS OF INTEREST}

Panli Zuo and Ignacio Vallines are employees of Siemens Healthcare China. Benjamin Schmitt is an employee of Siemens Healthcare Australia.

\section{REFERENCES}

1. Louis DN, Ohgaki H, Wiestler OD, Cavenee WK, Burger 
PC, Jouvet A, Scheithauer BW, Kleihues P. The 2007 WHO classification of tumours of the central nervous system. Acta Neuropathol. 2007; 114:97-109.

2. Johannessen AL, Torp SH. The clinical value of Ki-67/ MIB-1 labeling index in human astrocytomas. Pathol Oncol Res. 2006; 12:143-147.

3. Zhou J, Lal B, Wilson DA, Laterra J, van Zij1 PC. Amide proton transfer (APT) contrast for imaging of brain tumors. Magn Reson Med. 2003; 50:1120-1126.

4. Zhou J, Payen JF, Wilson DA, Traystman RJ, van Zijl PC. Using the amide proton signals of intracellular proteins and peptides to detect $\mathrm{pH}$ effects in MRI. Nat Med. 2003; 9:1085-1090.

5. Zhou J, van Zijl PC. Chemical exchange saturation transfer imaging andspectroscopy. Prog Nucl Magn Reson Spectrosc. 2006; 48:109-136.

6. Zhou J, Zhu H, Lim M, Blair L, Quinones-Hinojosa A, Messina SA, Eberhart CG, Pomper MG, Laterra J, Barker PB, van Zijl PC, Blakeley JO. Three-dimensional amide proton transfer MR imaging of gliomas: Initial experience and comparison with gadolinium enhancement. J Magn Reson Imaging. 2013; 38:1119-1128.

7. Togao O, Yoshiura T, Keupp J, Hiwatashi A, Yamashita K, Kikuchi K, Suzuki Y, Suzuki SO, Iwaki T, Hata N, Mizoguchi M, Yoshimoto K, Sagiyama K, et al. Amide proton transfer imaging of adult diffuse gliomas: correlation with histopathological grades. Neuro Oncol. 2014; 16:441448.

8. Park JE, Kim HS, Park KJ, Kim SJ, Kim JH, Smith SA. Pre- and posttreatment glioma: comparison of amide proton transfer imaging with MR spectroscopy for biomarkers of tumor proliferation. Radiology. 2016; 278:514-523.

9. Park JE, Kim HS, Park KJ, Choi CG, Kim SJ. Histogram analysis of amide proton transfer imaging to identify contrast-enhancing low-grade brain tumor that mimics high-grade tumor: increased accuracy of $\mathrm{mr}$ perfusion. Radiology. 2015; 277:151-161.

10. Sugahara T, Korogi Y, Kochi M, Ikushima I, Shigematu Y, Hirai T, Okuda T, Liang L, Ge Y, Komohara Y, Ushio Y, Takahashi M. Usefulness of diffusion-weighted MRI with echo-planar technique in the evaluation of cellularity in gliomas. J Magn Reson Imaging. 1999; 9:53-60.

11. Kang Y, Choi SH, Kim YJ, Kim KG, Sohn CH, Kim JH, Yun TJ, Chang KH. Gliomas: histogram analysis of apparent diffusion coefficient maps with standard- or highb-value diffusion-weighted MR imaging - correlation with tumor grade. Radiology. 2011; 261:882-890.

12. Lam WW, Poon WS, Metreweli C. Diffusion MR imaging in glioma: does it have any role in the pre-operation determination of grading of glioma? Clin Radiol. 2002; 57: 219-225.

13. Sadeghi N, D'Haene N, Decaestecker C, Levivier M, Metens T, Maris C, Wikler D, Baleriaux D, Salmon I, Goldman S. Apparent diffusion coefficient and cerebral blood volume in brain gliomas: relation to tumor cell density and tumor microvessel density based on stereotactic biopsies. AJNR Am J Neuroradiol. 2008; 29:476-482.

14. Jiang R, Jiang J, Zhao L, Zhang J, Zhang S, Yao Y, Yang S, Shi J, Shen N, Su C, Zhang J, Zhu W. Diffusion kurtosis imaging can efficiently assess the glioma grade and cellular proliferation. Oncotarget. 2015; 6:42380-42393. doi: 10.18632/oncotarget.5675.

15. Jahng GH, Li KL, Ostergaard L, Calamante F. Perfusion magnetic resonance imaging: a comprehensive update on principles and techniques. Korean J Radiol. 2014; 15:554557.

16. Roy B, Awasthi R, Bindal A, Sahoo P, Kumar R, Behari S, Ojha BK, Husain N, Pandey CM, Rathore RK, Gupta RK. Comparative evaluation of 3-dimensional pseudocontinuous arterial spin labeling with dynamic contrast-enhanced perfusion magnetic resonance imaging in grading of human glioma. J Comput Assist Tomogr. 2013; 37:321-326.

17. Zhou J, Hong X, Zhao X Gao JH, Yuan J. APT-weighted and NOE-weighted image contrasts in glioma with different RF saturation powers based on magnetization transfer ratio asymmetry analyses. Magn Reson Med. 2013; 70:320-327.

18. Griffiths JR. Are cancer cells acidic? Br J Cancer. 1991; 64:425-427.

19. Di Costanzo A, Scarabino T, Trojsi F, Giannatempo GM, Popolizio T, Catapano D, Bonavita S, Maggialetti N, Tosetti M, Salvolini U, d'Angelo VA, Tedeschi G. Multiparametric 3T MR approach to the assessment of cerebral gliomas: tumor extent and malignancy. Neuroradiology. 2006; 48:622-631.

20. Le Bihan D, Breton E, Lallemand D, Aubin ML, Vignaud J. Laval-Jeantet M. Separation of diffusion and perfusion in intravoxel incoherent motion MR imaging. Radiology. 1988; 168:497-505.

21. Le Bihan D, Breton E, Lallemand D, Grenier P, Cabanis E, Laval-Jeantet M. MR imaging of intravoxel incoherent motions: application to diffusion and perfusion in neurologic disorders. Radiology. 1986; 161:401-407.

22. Bai Y, Lin Y, Tian J, Shi D, Cheng J, Haacke EM, Hong X, Ma B, Zhou J, Wang M. Grading of gliomas by using monoexponential, biexponential, and stretched exponential diffusion-weighted MR imaging and diffusion kurtosis MR imaging. Radiology. 2016; 278:496-504.

23. Penfield JG, Reilly RF Jr. What nephrologists need to know about gadolinium. Nat Clin Pract Nephrol. 2007; 3:654-668.

24. Akkus Z, Sedlar J, Coufalova L, Korfiatis P, Kline TL, Warner JD, Agrawal J, Erickson BJ. Semi-automated segmentation of pre-operative low grade gliomas in magnetic resonance imaging. Cancer Imaging. 2015; 15:12.

25. Huang D, Wu B, Shi K, Ma L, Cai Y, Lou X. Reliability of three-dimensional pseudo-continuous arterial spin labeling MR imaging for measuring visual cortex perfusion on two 3T scanners. PLoS One. 2013; 8:e79471. 